\title{
Editorial
}

\section{Optical Characterization of Nanomaterials}

\author{
Mun Seok Jeong, ${ }^{1}$ Gon Namkoong, ${ }^{2}$ Clare C. Byeon, ${ }^{3}$ Jong Su Kim, ${ }^{4}$ and Hong Seok Lee ${ }^{5}$ \\ ${ }^{1}$ Department of Energy Science, Center for Integrated Nanostructure Physics, Institute for Basic Science, Sungkyunkwan University, \\ Suwon 440-746, Republic of Korea \\ ${ }^{2}$ Department of Electrical and Computer Engineering, Old Dominion University, Norfolk, VA 23529, USA \\ ${ }^{3}$ School of Mechanical Engineering, Kyungpook National University, Daegu 702-701, Republic of Korea \\ ${ }^{4}$ Department of Physics, Yeungnam University, Gyeongsan 712-749, Republic of Korea \\ ${ }^{5}$ Department of Physics, Jeju National University, Jeju 690-756, Republic of Korea
}

Correspondence should be addressed to Mun Seok Jeong; mjeong@skku.edu

Received 13 June 2014; Accepted 13 June 2014; Published 24 July 2014

Copyright (c) 2014 Mun Seok Jeong et al. This is an open access article distributed under the Creative Commons Attribution License, which permits unrestricted use, distribution, and reproduction in any medium, provided the original work is properly cited.

Studies on nanomaterials have recently been carried out widely and intensively in many fields in order to explore the new era of the nanoscopic world and to overcome the limitation of typicalness of the bulk materials. Nanomaterials with the enhanced mechanical, electrical, and optical properties distinctively different from the bulk material properties allow us to surmount many areas that the conventional technology could not address. Nowadays, such properties of the nanomaterials may be characterized electrically and mechanically by direct contact with the nanomaterials. Meanwhile, the optical methods take advantage of observing the essential characteristics of the nanomaterials without significantly modifying or permanently damaging them due to their noncontact and noninvasive nature. Common optical characterization methods include absorbance/transmittance, photoluminescence, and Raman scattering measurement.

This special issue arranges a series of up-to-date articles dealing with characterizing the various nanomaterials by using such optical characterization methods. M. Benson et al. discovers, from the absorbance analysis, the metamaterial properties of silver nanorod thin film grown by oblique angle deposition, "Optical characterization of silver nanorod thin films grown using oblique angle deposition." Y. C. Choi et al. describes a purity analysis for carbon nanotubes by using Raman scattering, "Novel method of evaluating the purity of multiwall carbon nanotubes using Raman spectroscopy." $\mathrm{N}$. Shanmugam et al. reports the effect of thermal process on ZnS nanocrystals synthesized by chemical precipitation by using UV-Vis absorption and photoluminescence, "Effect of annealing on the ZnS nanocrystals prepared by chemical precipitation method." By FT-IR, absorbance, and fluorescence spectroscopy, T. L. Tan et al. observe the change in optical characteristics of $\mathrm{Mn}$-doped $\mathrm{ZnO}$ nanoparticles synthesized by coprecipitation method while varying the manganese dopant concentration, "Tunable band gap energy of Mn-doped $\mathrm{ZnO}$ nanoparticles using the coprecipitation technique." F. Severiano et al. introduces an electroluminescent device based on white light emission from $\mathrm{ZnO}$ :In/PS by electroluminescence and photoluminescence, "Electroluminescent devices based on junctions of indium doped zinc oxide and porous silicon." By using photoluminescence, H.-S. Chin and L.-S. Chao investigate the thermal annealing effect on $\mathrm{ZnO}$ thin film grown by radio frequency sputtering, "The effect of thermal annealing processes on structural and photoluminescence of zinc oxide thin film." Measuring the optical transmittance, F.-H. Wang et al. analyzes the hydrogen effect on sputterdeposited $\mathrm{Al}_{2} \mathrm{O}_{3}$-doped $\mathrm{ZnO}$ thin films, "Effects of hydrogen on the optical and electrical characteristics of the sputterdeposited $\mathrm{Al}_{2} \mathrm{O}_{3}$-doped $\mathrm{ZnO}$ thin films." T. K. Kim et al. observes the negative differential resistance from molecular nanosilver chain, "Symmetric negative differential resistance in a molecular nanosilver chain." F. Q. Zhou et al. proposes single ring-shaped nanotubes, dimmers, and arrays and discuss their optical transmission properties, "Formation and evolution mechanism of plasmon resonance from single ringshaped nanotube to dimer and arrays." 
As we have seen from the papers in this special issue, the optical characterization methods are essential in analyzing the various fundamental and functional properties of the nanomaterials. The noncontact and noninvasive nature makes the optical characterization methods one of the best ideal and at the same time practical tools to investigate and to take advantage of many different types of nanomaterials. We believe that the articles in this special issue would serve as excellent examples of how these optical methods are utilized in characterization of some of the well-known nanomaterials.

Mun Seok Jeong

Gon Namkoong

Clare C. Byeon

Jong Su Kim

Hong Seok Lee 

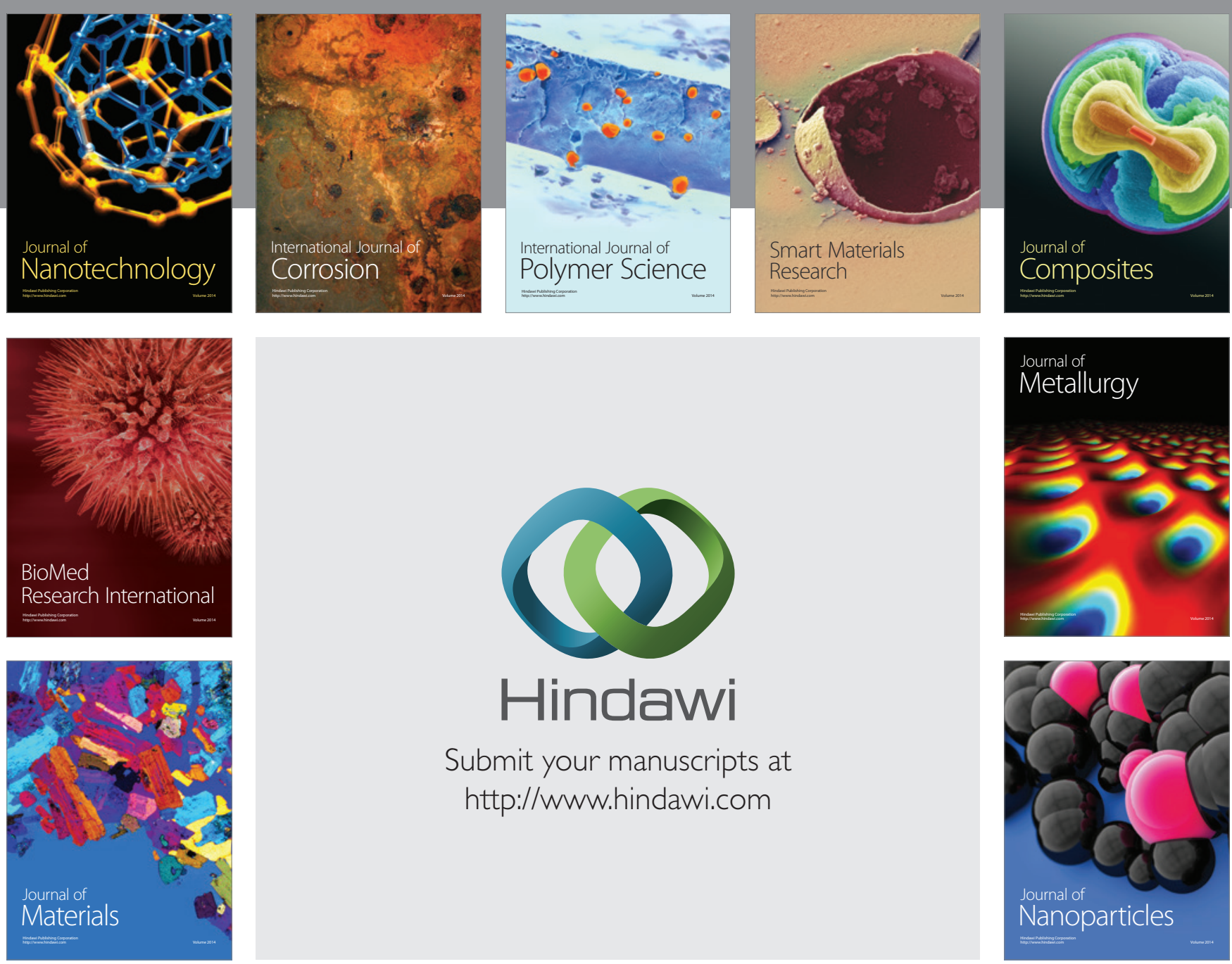

Submit your manuscripts at http://www.hindawi.com
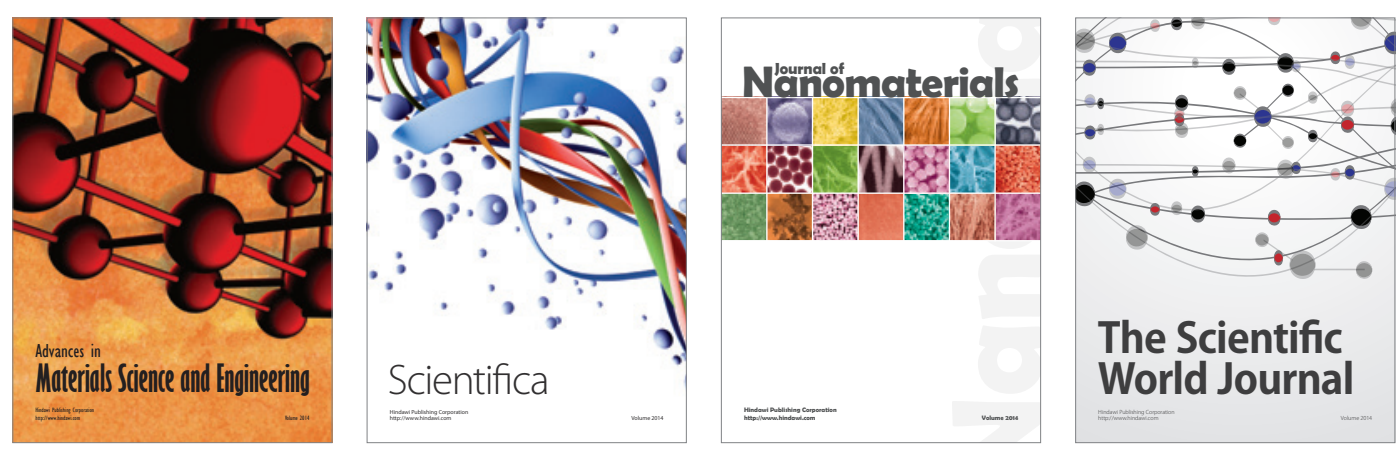

\section{The Scientific World Journal}
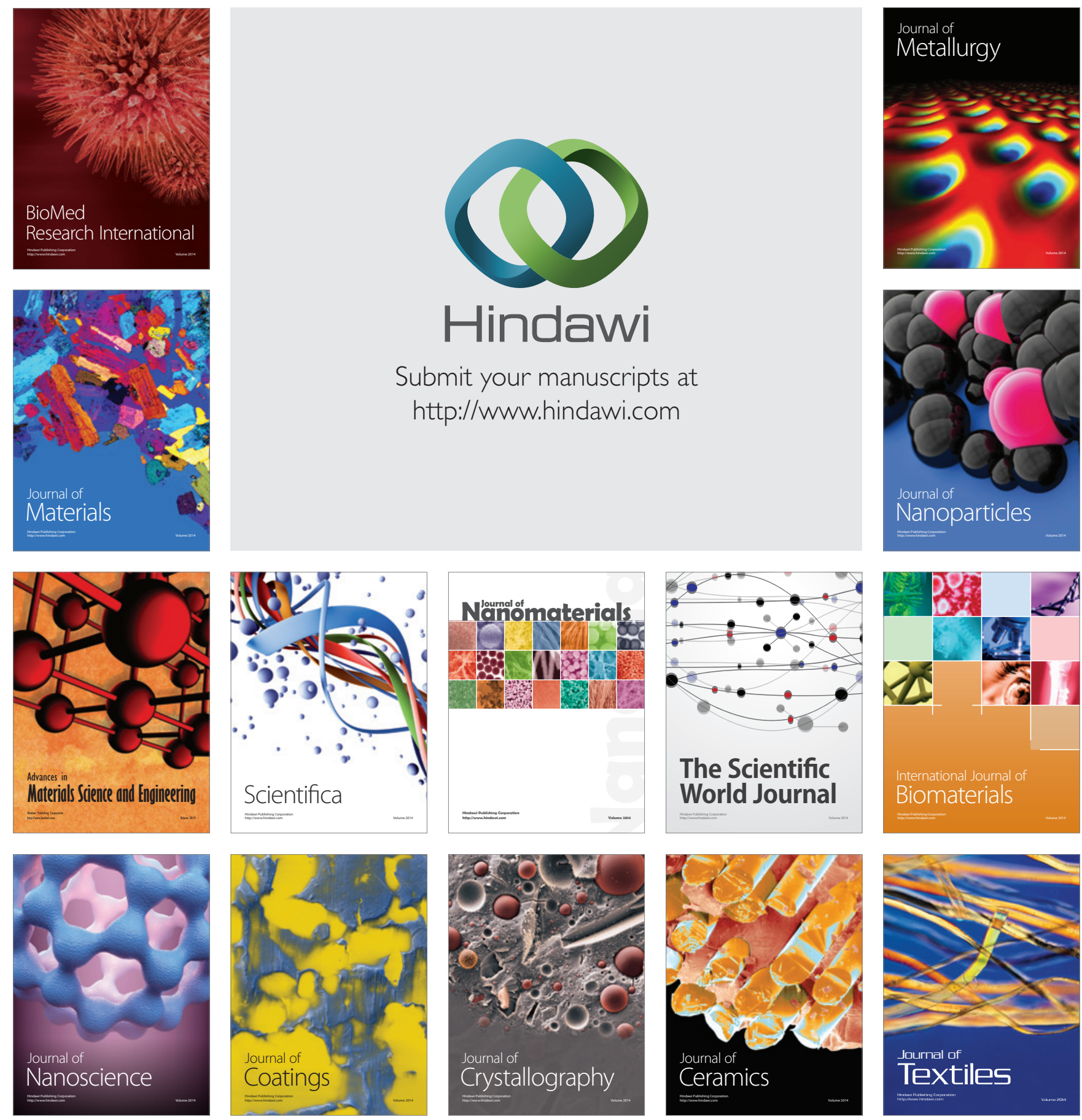